\title{
Short communication: Changes under low ambient temperatures in the milk lipodome and metabolome of mid-lactation cows after dehorning as a calf
}

\author{
T. Baars, ${ }^{1 *}$ G. Jahreis, ${ }^{2,3}$ S. Lorkowski, ${ }^{3,4}$ C. Rohrer,,${ }^{3,4}$ J. Vervoort, ${ }^{5}$ and K. Hettinga ${ }^{6}$ \\ ${ }^{1}$ Research Institute for Organic Agriculture (FiBL), Ackerstrasse, 5070 Frick, Switzerland \\ ${ }^{2}$ Department of Nutritional Physiology, Institute of Nutrition, Friedrich Schiller University Jena, Dornburger Straße 24, 07743 Jena, Germany \\ ${ }^{3}$ Competence Cluster for Nutrition and Cardiovascular Health (nutriCARD), Halle-Jena-Leipzig, Dornburger Straße 25, 07743 Jena, Germany \\ ${ }^{4}$ Department of Nutritional Biochemistry and Physiology, Institute of Nutrition, Friedrich Schiller University Jena, Dornburger Straße 25, \\ 07743 Jena, Germany \\ ${ }^{5}$ Laboratory of Biochemistry, Wageningen University, Dreijenlaan 3, 6703 HA Wageningen, the Netherlands \\ ${ }^{6}$ Food Quality and Design Group, Wageningen University, PO Box 17, $6700 \mathrm{AH}$ Wageningen, the Netherlands
}

\section{ABSTRACT}

Horns are living tissue and cows can use their horns for thermoregulatory purposes. We investigated the effect of the presence of horns on the metabolome of milk serum and lipidome of milk fat, to assess the physiological effect of dehorning. Milk sampling took place at low ambient temperatures of -6 to $2^{\circ} \mathrm{C}$. Horned and dehorned cows were kept in a mixed herd of Holstein Friesian and Brown Swiss cows. The hypothesis was that horned cows needed to increase their metabolism to compensate for additional heat loss through the presence of their horns. No differences were observed in milk yield, milk solids, and somatic cell counts between horned and dehorned cows. For the milk metabolome, horned cows showed an upregulation of several glucogenic AA that could be transformed into glucose for energy supply and a downregulation of sugar intermediates and $\gamma$-glutamylcysteine compared with dehorned cows. The fatty acid (FA) composition in horned cows showed a shift toward decreased odd medium-chain FA (C7:0, C9:0, and C11:0) and increased cis-vaccenic acid (C18:1n-7 cis-11) and stearidonic acid (C18:4n-3). The changes in milk composition related to additional heat loss in horned cows indicate a competition in C3 metabolism for glucose synthesis and de novo FA synthesis under cold stress.

Key words: dehorning, ruminant physiology, lipodome, metabolome, cold stress

\section{Short Communication}

Horns in Bovidae differ in their morphology and size. A nonliving keratin horn sheath covers the living,

Received July 23, 2018.

Accepted December 5, 2018.

*Corresponding author: t.baars@fingerprint.nl vascularized bony core as an extension of the frontal bone sinuses (Hoefs, 2000). Besides defense, the horns of cows play a role in the body and brain temperature homeostasis (Taylor, 1966). Homeothermic endotherm animals follow different heat conservation strategies to adapt to the ambient temperature and different body parts are involved (Henning et al., 2018). The horn provides most Artiodactyls (even-toed ungulates) the means of selectively cooling the brain (Taylor, 1966) through the carotid rete located under the brain, offering the possibility of counter-current cooling (O'Brien et al., 2016). Both horns and hoofs contain highly vascular tissues (Zerpa et al., 2010) and vasoconstriction or vasodilation of the vessel walls is responsible for the thermoregulatory capacities of the horn. Although cow horns are small compared with the animal's surface area, horns contribute significantly to the total noninsulated areas, measurable through infrared thermography (Kim et al., 2014). For male Canadian Barbary sheep, Picard et al. (1994) calculated a heat loss through the horns of 7 to $10 \%$ of the total energy budget. At an environmental temperature below $4^{\circ} \mathrm{C}$, the temperature of horns remained at a constant level (Kim et al., 2014). The horn blood circulation prevents frostbite and starvation of the inner horn tissues (Taylor, 1966). Brouček et al. (1991) distinguished 3 major physiological responses to cold stress: (1) increased metabolic heat production, (2) higher cardiac output combined with redistribution of blood flow, and (3) mobilization of substrates for metabolism: glucose, AA, and fatty acids (FA). The amount of energy from complete AA catabolism is low and involves only 10 to $15 \%$ of total energy expenditure in bovine. According to Early et al. (1990), the increased pool size and turnover rate of glucose is an adaptation to low ambient temperatures. The principal energy substrates, however, would be lipids or VFA. Based on their gene expression, cows under severe cold stress showed clear changes in lipid metabolism (Xu et al., 2017). 
In the current cross-sectional study, we investigated the differences in metabolism due to differences in heat loss, depending on the competition in metabolic pathways for either milk production or maintenance of body temperature in horned and dehorned cows. The milk serum metabolite profile and milk FA composition were analyzed of $2 \times 28$ paired cows. Horned and dehorned cows within 2 breeds of cows, Holstein Friesian (HF) and Brown Swiss (BS), were kept in a mixed group of 300 animals. One fodder place per cow was present. Cows were fed hay ad libitum and twice a day, cows were fixed on the fodder track and fed an additional small amount of concentrates (based on a mixture of grains, chopped beet roots, and legumes). The calculated amount of hay was $85 \%$ of their DMI. No measurements could be made on individual feed intake, which is a weak part of the study. Due to missing information about the individual DMI per cow, effects of differences in intake between horned and dehorned cows cannot be excluded.

On 3 occasions, evening milk was sampled from paired horned and dehorned cows. In November and December 2013, the same group of cows was sampled. In the next lactation (March 2015), there was some overlap with cows sampled earlier. Environmental temperatures and climate data were taken from surrounding weather stations. The day before milk sampling, minimum temperatures were $+2.2,-2.5$, and $-6.5^{\circ} \mathrm{C}$, respectively. The farm climate is continental and showed a $30-\mathrm{yr}$ average minimum temperature of $0.6,-2.5,-5.1,-5.4$, and $-2.1^{\circ} \mathrm{C}$ (November to March). At milk sampling, we had moderately cold ambient temperatures at the lower end of acceptability for lactating cows (Angrecka and Herbut, 2015). Under low ambient temperatures it is expected that horned cows need to increase their metabolism at a higher rate compared with dehorned cows.

Milk was stored at $-18^{\circ} \mathrm{C}$. For the FA analysis, frozen samples were thawed at room temperature and freeze-dried. The milk powder was used for Soxhlet extraction. Fatty acid methyl esters were prepared using $\mathrm{NaOCH}_{3}$. Gas chromatography procedures were used to resolve all FA as described by Kuhnt et al. (2011). Identification of FA was based on internal standards; for quantification the peak areas were related to the sum of all identified peaks (proportion of FA of total identified FAME). To determine the metabolite profile of the milk serum, ${ }^{1} \mathrm{H}-\mathrm{NMR}$ metabolomics was performed as described in detail by Lu et al. (2013). The peak area of each assignment is relative to the calibration standard 3-trimethylsilyl-2,2,3,3-tetradeuteropropionate, resulting in a relative peak area in arbitrary units that was used for statistical analyses.
Data were analyzed in a linear mixed model using SPSS Version 20 (IBM Corp., Armonk, NY). Milk control data remained untransformed, only SCC were transformed in natural logarithm. Fatty acids were natural-logarithm transformed if necessary due to kurtosis of the data. Relative peak areas of metabolites were $\log _{2}$ transformed. Samples that were incorrectly coded ( $\mathrm{n}=1$ pair) and outliers, defined as having $>5 \%$ of their FA being in the extreme $10 \%$ of the individual FA concentrations, were excluded from further analysis ( $\mathrm{n}=2$ pairs). In the linear mixed model $(\mathrm{n}=50)$, the cow ID was taken as a subject and date of sampling as a repeated value. The model was built on the fixed factors: horn status (horned and dehorned), breed (HF and BS), and age class [lactation number smaller or equal than $2(\leq 2)$, and larger or equal than $3(\geq 3)]$. The interactions of horn $\times$ breed, and horn $\times$ age were integrated in the model. The covariance effect on significant FA and metabolites was tested for the factor horn after integration of milk fat content and fat- and protein-corrected milk yield $(\mathrm{kg})$ as single covariates. The level of significance was taken at $5 \%(P<0.05)$. Solely the effects of horn status, also in the single breeds, will be discussed here. No differences were present in basic milk features depending on horn status (Table 1). This indicates that feed intake was similar between the 2 groups of animals and not affected by possible competition through the presence of horns. Horn status had an effect on both AA and FA metabolism (Tables 2 and 3). The increased level of creatinine $(P<0.074)$ in horned cows indicates higher muscle metabolic activity, in accordance with a higher muscle shivering (Bell et al., 1974). This coincides with the upregulation of several AA (cystine, glycine, proline, and kynurenine) in horned cows. Kynurenine can be both glucogenic and ketogenic AA. Cystine, glycine, and proline are glucogenic, acting as precursors in the glucose synthesis pathway. The lower levels of $\gamma$-glutamyl-cysteine in horned cows might be due to competition for ATP in gluconeogenesis and glutathione synthesis.

Small differences were observed in the horn status between the 2 breeds. Horned BS cows showed an upregulation of glyoxylic acid, creatinine, and $\alpha$-ketoglutarate compared with dehorned BS cows. Glyoxylic acid is an intermediate of the glyoxylate cycle, where in the process of $\beta$-oxidation, FA are transformed into acetate that can be further transformed into glucose. However, the presence of the glyoxylate shunt in mammals is still under discussion (Kunze et al., 2006). This implies that horned BS cows may depend on additional FA oxidation and glucose formation for their energy supply. In contrast and in comparison to dehorned HF cows, horned HF cows showed decreased $\alpha$-ketoglutarate and 
Table 1. Means and SD of horned $(\mathrm{H})$ and dehorned (D) cows and the level of significance ( $P$-value) plus interactions with breed $(\mathrm{B})$ and age $(\mathrm{A})^{1}$

\begin{tabular}{lccccc}
\hline Feature & \multicolumn{1}{c}{$\mathrm{H}$} & $\mathrm{D}$ & $\mathrm{H}$ & $\mathrm{H} \times \mathrm{B}$ & $\mathrm{H} \times \mathrm{A}$ \\
\hline Date of calving & Nov. 13, 2013 $(3.0)$ & Nov. 16, 2013 $(6.2)$ & 0.848 & & \\
Lactation number & $2.58(1.30)$ & $3.15(1.35)$ & 0.819 & & \\
DIM & $161.2(57.5)$ & $158.3(58.9)$ & 0.195 & 0.275 & 0.291 \\
Milk (kg) & $22.1(5.0)$ & $22.7(4.2)$ & 0.576 & 0.955 & 0.321 \\
Fat $(\%)$ & $4.21(0.50)$ & $4.33(0.87)$ & 0.578 & 0.284 & 0.089 \\
Protein (\%) & $3.55(0.35)$ & $3.56(0.35)$ & 0.866 & 0.712 & 0.815 \\
FPCM (kg) & $22.9(4.5)$ & $24.1(5.0)$ & 0.741 & 0.678 & 0.743 \\
lnSCC & $3.77(1.00)$ & $3.95(0.74)$ & 0.469 & 0.780 & 0.093 \\
Urea (\%) & $172(94)$ & $178(106)$ & 0.058 & 0.637 & 0.517 \\
Lactose (\%) & $4.76(0.17)$ & $4.77(0.17)$ & 0.130 & 0.501 & 0.122 \\
Lactose (kg) & $1.05(0.25)$ & $1.09(0.21)$ & 0.585 & 0.618 & 0.775 \\
\hline
\end{tabular}

${ }^{1}$ Results based on a mixed model with exception for date of calving and number of lactation (based on a nonparametric test). FPCM = fat- and protein-corrected milk yield; $\ln =\log$ normal.

urea concentrations, possibly due to the transamination to glutamate. Uridine diphosphate (UDP)-hexose was lower in horned HF cows, whereas $N$-acetyl-UDPgalactose was lower in horned animals of both breeds. These sugar metabolites belong to the lactose synthesis pathway, although no differences in lactose content and lactose yield were found (Table 1). The lower concentrations of urea in horned HF cows indicate a lower gluconeogenesis via AA. This could lead to a lower availability of glucose in the udder, reflected in the changes in sugar metabolites. Compared with horned BS cows, in horned HF cows the process of gluconeogenesis is in a stronger competition with lactose synthesis and both pathways deal with the same C3 intermediates.

The milk FA composition showed several minor differences between horned and dehorned cows with no or limited interactions with breed and age. Two n-3 FA, $\alpha$-linolenic acid (C18:3n-3) and stearidonic acid (C18: $4 \mathrm{n}-3)$, were higher in horned cows, although $\alpha$-linolenic acid was only higher in the older horned cows (parity
$>3$ ). These long-chain PUFA originate from the cow's dietary intake of lipids and become largely biohydrogenated in the rumen. A decrease of the odd-chain SFA C7:0, C9:0, C11:0, and C13:0 also occurs in horned cows (Table 3). The shift in FA composition between horned and dehorned cows is in accordance with an earlier study (Wohlers, 2011), where a similar upregulation of long-chain PUFA plus a downregulation of mediumchain SFA was found. Up to C16:0, milk FA are de novo synthesized via VFA (C2, C3, and $\mathrm{C} 4)$ in the cow's udder (Vlaeminck et al., 2006), in which C3 is being used for the synthesis of odd-chain FA. For the milk FA synthesis, the supply of $\mathrm{C} 2$ and $\mathrm{C} 4 \mathrm{VFA}$ is dominant, whereas C3 for FA synthesis competes with the use of C3 for gluconeogenesis (Fulco, 1983). In horned cows, 2 branched-chain FA, C15 iso and C17 iso, are slightly higher, but significance for C15 iso was only reached in the older cows (parity $\geq 3$ ). Finally, cis-vaccenic acid $(\mathrm{C} 18: 1 \mathrm{n}-7$ cis-11) is upregulated in horned cows, which might originate from the adipose tissue (Kelly et al.,

Table 2. Significant metabolites: the $\log _{2}$ mean for horned $(\mathrm{H})$ and dehorned $(\mathrm{D})$ cows, SEM, the level of significance $(P$-value) for horn status, and the interactions with breed and age class ${ }^{1}$

\begin{tabular}{|c|c|c|c|c|c|c|c|c|c|c|c|}
\hline Metabolite & \multicolumn{4}{|c|}{ Horn status ( $\log _{2}$ mean and SEM) } & \multicolumn{7}{|c|}{$P$-value } \\
\hline Cystine & 13.67 & 0.20 & 12.97 & 0.21 & 0.000 & 0.270 & 0.954 & & & & \\
\hline Kynurenine & 14.01 & 0.17 & 13.48 & 0.15 & 0.037 & 0.883 & 0.574 & & & & \\
\hline Proline & 21.32 & 0.09 & 21.10 & 0.09 & 0.001 & 0.151 & 0.219 & & & & \\
\hline $\mathrm{N}$-Acetyl UDP-galactose & 15.65 & 0.22 & 15.87 & 0.20 & 0.000 & 0.444 & 0.883 & & & & \\
\hline$\alpha$-Ketoglutaric acid & 16.94 & 0.12 & 16.86 & 0.18 & 0.162 & 0.004 & 0.042 & 0.011 & 0.010 & 0.000 & 0.006 \\
\hline UDP-hexose & 17.90 & 0.15 & 18.28 & 0.10 & 0.190 & 0.008 & 0.062 & 0.002 & 0.654 & & \\
\hline Urea & 18.26 & 0.19 & 18.76 & 0.10 & 0.057 & 0.017 & 0.055 & 0.002 & 0.477 & & \\
\hline
\end{tabular}

${ }^{1}$ Only $P$-values are shown for single factors, when the outcome was significant. $\mathrm{B}=$ breed; $\mathrm{A}=$ age class; $\mathrm{H}$ HF $=$ horned Holstein Friesian; $\mathrm{H}$ BS $=$ horned Brown Swiss; $\mathrm{H}<2=$ horned in age class equal or less than 2 lactations; $\mathrm{H}>3=$ horned in age class equal or greater than 3 lactations. UDP $=$ uridine diphosphate. 
Table 3. Significant single fatty acids (FA; g/100 g): the mean and SEM for horned (H) and dehorned (D) cows, the level of significance $\left(P\right.$-value) for horn status, and the interactions with breed and age class ${ }^{1}$

\begin{tabular}{|c|c|c|c|c|c|c|c|c|c|}
\hline \multirow[b]{2}{*}{ Single FA (g/100 g) } & \multicolumn{4}{|c|}{ Horn status (mean and SEM) } & \multicolumn{5}{|c|}{$P$-value } \\
\hline & $\mathrm{H}$ & SEM & $\mathrm{D}$ & SEM & $\mathrm{H}$ & $\mathrm{H} \times \mathrm{B}$ & $\mathrm{H} \times \mathrm{A}$ & $\mathrm{H}<2$ & $\mathrm{H}>3$ \\
\hline C9:0 & 0.031 & 0.001 & 0.037 & 0.002 & 0.001 & 0.437 & 0.242 & & \\
\hline C11:0 & 0.060 & 0.002 & 0.072 & 0.003 & 0.001 & 0.148 & 0.084 & & \\
\hline C13:0 & 0.107 & 0.003 & 0.117 & 0.004 & 0.010 & 0.107 & 0.017 & 0.002 & \\
\hline C15 iso & 0.313 & 0.008 & 0.291 & 0.008 & 0.143 & 0.338 & 0.027 & & 0.006 \\
\hline $\mathrm{C} 16: 4(\mathrm{n}-1)$ & 0.279 & 0.006 & 0.259 & 0.006 & 0.037 & 0.381 & 0.289 & & \\
\hline$\alpha-\mathrm{C} 18: 3$ cis- 9, cis- 12, cis-15 (n-3) & 0.741 & 0.025 & 0.671 & 0.025 & 0.146 & 0.119 & 0.027 & & 0.035 \\
\hline $\mathrm{C} 18: 4$ cis-6,cis-9,cis-12,cis-15 (n-3) & 0.018 & 0.001 & 0.015 & 0.001 & 0.050 & 0.781 & 0.348 & & \\
\hline
\end{tabular}

${ }^{1}$ Only $P$-values for single factors are shown, if the outcome was significant. $\mathrm{B}=$ breed; $\mathrm{A}=$ age class; $\mathrm{H}<2=$ horned in age class equal or less than 2 lactations; $\mathrm{H}>3=$ horned in age class equal or greater than 3 lactations.

2013). Ma et al. (2015) showed that the level of lipid desaturation is an answer in cells to adapt to different environmental temperatures. Increased levels of C18: $1 \mathrm{n}-7$ were found in roundworms in the cold, but this physiological adaptation is genetically expressed in all mammals. Their results were in accordance with the higher C18:1n-7 levels found in milk from horned cows. Horned cows expressed the additional need to maintain the cell membrane fluidity in the cold.

The decrease in metabolites of the lactose pathway, as well as the drop in $\mathrm{C} 3$-derived FA, indicate a reduction in ruminal C3 production in horned cows. The C3 pathways for milk production and body temperature maintenance become competitive in the cold-stressed horned cows. Cold exposure may change rumen metabolism of FA, but also increases the passage of feed through the rumen (Kennedy and Milligan, 1978). The increased passage rate may also have reduced the biohydrogenation of long-chain PUFA, causing increased levels of these PUFA in horned cows (Table 3). Although FA composition in milk is generally mainly influenced by feed, the higher heat demand in horned cows additionally affects the lipid metabolism in the rumen, thereby causing the changes in FA seen in this study. For most of the FA, the quantitative differences are in a similar size order as the differences between a summer and winter diet at this farm (unpublished data). This indicates that the effect seen in Table 3 is too large to be explained by the differences in feed intake alone.

After a covariance analysis to determine whether findings were influenced by the milk fat content or fatand protein-corrected milk yield of single cows, several metabolites and FA (saturated odd medium-chain FA, cis-vaccenic acid, cystine, glycine, and kynurenine) remained significantly different.
Although we cannot exclude the possibility that differences in feed intake between the paired cows exist, the outcomes of this study can be understood and explained through the knowledge about additional heat loss through horns under low ambient temperatures (Taylor, 1966; Zerpa et al., 2010). At a physiological level, under low ambient temperatures, horn status affected the competition for glucose for either heat (ATP) or milk production (lactose), both pathways based on the competition of C3 metabolites, which may have caused reduced synthesis of odd short-chain FA in horned cows. Horned HF and BS cows used slightly different metabolic pathways to control their higher energy demand. Horned BS cows demonstrated increased FA catabolism, probably due to their overall higher body mass score, and this was also reflected in changes in their gene expression as found by Xu et al. (2017). In adapted wild animals, the reduced size of the vascular bed, relatively thick keratin sheath, and curled position of horns act to reduce heat loss in cold environments (Picard et al., 1999). We have no information on differences in horn morphology between the breeds, but differences in horn morphology might further explain the difference in metabolic pathways we found between the 2 breeds.

In conclusion, in an on-farm experiment, in which milk from horned and dehorned cows was compared on their lipodome and metabolome, no differences were present in milk yield and gross composition. Low ambient temperatures at the time of sampling may have led to a higher energy demand of horned cows due to compensation for additional heat radiation through their horns. Markers for horned cows under low ambient temperatures were several odd medium-chain FA (C7:0-C13:0), C18:1n-7 cis-11, and several glucogenic AA (cystine, glycine, and proline). 


\section{ACKNOWLEDGMENTS}

We are grateful for the financial support of Software AG Stiftung, Darmstadt, Germany, which made this work possible. We thank the co-workers from Juchowo farm, Silnowo, Poland, for their support and collection of the milk samples.

\section{REFERENCES}

Angrecka, S., and P. Herbut. 2015. Conditions for cold stress development in dairy cattle kept in free stall barn during severe frosts. Czech J. Anim. Sci. 60:81-87.

Bell, A. W., G. E. Thompson, and J. D. Findlay. 1974. The contribution of the shivering hind leg to the metabolic response to cold of the young ox (Bos taurus). Pflugers Arch. 346:341-350.

Brouček, J., M. Letkovicová, and K. Kovalcuj. 1991. Estimation of cold stress effect on dairy cows. Int. J. Biometeorol. 35:29-32.

Early, R. J., J. R. Thompson, and R. J. Christopherson. 1990. Glucose and alanine metabolism in chronically cold-exposed sheep. Can. J. Anim. Sci. 70:517-524.

Fulco, A. J. 1983. Fatty acid metabolism in bacteria. Prog. Lipid Res. $22: 133-160$.

Henning, B., B. de Sá Carvalho, J. L. Boldrini, S. F. dos Reis, and D. O. V. de Andrade. 2018. Statistical estimation of surface heat control and exchange in endotherms. Open J. Stat. 8:220.

Hoefs, M. 2000. The thermoregulatory potential of Ovis horn cores. Can. J. Zool. 78:1419-1426.

Kelly, M. J., R. K. Tume, S. Newman, and J. M. Thompson. 2013. Genetic variation in fatty acid composition of subcutaneous fat in cattle. Anim. Prod. Sci. 53:129-133.

Kennedy, P. M., and L. P. Milligan. 1978. Effects of cold exposure on digestion, microbial synthesis and nitrogen transformations in sheep. Br. J. Nutr. 39:105-117.

Kim, N. Y., S. J. Kim, J. H. Park, M. R. Oh, S. Y. Jang, D. H. Kim, S. H. Sung, B. T. Jeon, and S. H. Moon. 2014. Seasonal changes in the body surface temperature of Hanwoo (Bos taurus coreanae) steers. Anim. Prod. Sci. 54:1476-1480.
Kuhnt, K., M. Baehr, C. Rohrer, and G. Jahreis. 2011. Trans fatty acid isomers and the trans-9/trans-11 index in fat containing foods. Eur. J. Lipid Sci. Technol. 113:1281-1292.

Kunze, M., I. Pracharoenwattana, S. M. Smith, and A. Hartig. 2006. A central role for the peroxisomal membrane in glyoxylate cycle function. Biochim. Biophys. Acta 1763:1441-1452.

Lu, J., E. Antunes Fernandes, A. E. Paez Cano, J. Vinitwatanakhun, S. Boeren, T. van Hooijdonk, A. van Knegsel, J. Vervoort, and K. A. Hettinga. 2013. Changes in milk proteome and metabolome associated with dry period length, energy balance, and lactation stage in postparturient dairy cows. J. Proteome Res. 12:3288-3296.

Ma, D. K., Z. Li, A. Y. Lu, F. Sun, S. Chen, M. Rothe, R. Menzel, F. Sun, and H. R. Horvitz.2015. Acyl-CoA dehydrogenase drives heat adaptation by sequestering fatty acids. Cell 161:1152-1163.

O'Brien, H. D., P. M. Gignac, T. L. Hieronymus, and L. M. Witmer 2016. A comparison of postnatal arterial patterns in a growth series of giraffe (Artiodactyla: Giraffa camelopardalis). PeerJ 4:e1696.

Picard, K. D. W. Thomas, M. Festa-Bianchet, F. Belleville, and A Laneville. 1999. Differences in the thermal conductance of tropical and temperate bovid horns. Ecoscience 6:148-158.

Picard, K., D. W. Thomas, M. Festa-Bianchet, and C. Lanthier. 1994. Bovid horns: An important site for heat loss during winter? J. Mammal. 75:710-713.

Taylor, C. R. 1966. The vascularity and possible thermoregulatory function of the horns in goats. Physiol. Zool. 39:127-139.

Vlaeminck, B., V. Fievez, S. Tamminga, R. J. Dewhurst, A. van Vuuren, D. De Brabander, and D. Demeyer. 2006. Milk odd- and branched-chain fatty acids in relation to the rumen fermentation pattern. J. Dairy Sci. 89:3954-3964.

Wohlers, J. 2011. Ermittlung geeigneter Methoden zur Differenzierung und Qualitätsbeurteilung unterschiedlicher Milchqualitäten aus verschiedenen on-farm-Experimenten. Dissertation, Univ. Kassel, Witzenhausen, Germany.

Xu, Q., Y. C. Wang, R. Liu, L. F. Brito, L. Kang, Y. Yu, D. S. Wang, H. J. Wu, and A. Liu. 2017. Differential gene expression in the peripheral blood of Chinese Sanhe cattle exposed to severe cold stress. Genet. Mol. Res. 20:16.

Zerpa, H., Y. Berhane, J. Elliott, and S. R. Bailey. 2010. The effect of cooling on the contractility of equine digital small lamellar arteries: Modulating role of the endothelium. Exp. Physiol. 95:1033-1042. 